\title{
Research on Networking Technology of Motor Transport Industry
}

\author{
Fahai $\mathbf{L i}^{1, a}$ \\ ${ }^{1}$ School of Electrical and Information Engineering, Hubei University of Automotive Technology, \\ Shiyan, China, 442002 \\ a sylfh73@163.com
}

\begin{abstract}
With highly attention paid to the transportation industry in our country and technology is widely used in automobile transport industry, the research which points out networking technology of automobile transportation industry can help reduce transport costs. In order to improve network management efficiency of automobile transport industry, we elaborate the key technology of transport industry on data-link network design, as well as classified networking technology. Furthermore, we made a deep research on joint and quit network antenna model simulation technology. Finally, we put forward an analysis that applying simulation techniques to transport industry networking technology.
\end{abstract}

\section{Keywords: Automobile Transport; Networking} Technology; OPNET

\section{Introduction}

With the high attention paid to the transportation industry in our country and technology is widely used in automobile transport industry, domestic and foreign software companies have made deeper and deeper research on the transport date network. All kinds of efficient transport data network appears one after another. Here are some issues in the research of automotive transportation network technology: first, the use of directional antennas, sender and receiver antenna must be aligned in order to complete the normal communication, it becomes extremely difficult when no adjacent node topology information found in the initial stage of neighbor. Second, motor transport data link point moves too faster, resulting in an unstable link, we need to design a new way of joint and quit network quickly.

\section{Key Technology Research on Transport Industry Data Link Network Design}

\subsection{Antenna technology}

Since isotropic antenna gain is low, and is suitable for lowfrequency, low-speed communication, and cannot provide additional anti-jamming capability and reduced detectability. But the narrow beam of directional antenna can produce a high gain, low side lobe characteristics. This can greatly improve the communication distance [1]. Due to various advantages of spray antennas, recently, many countries have also increased research efforts for a directional antenna.

\subsection{Antenna alignment and tracking algorithm design and selection}

There are merits and demerits in different antenna pointing control way, therefore they have their own applicable scope. In the initial period of system, no other node location information, due to loss of information for some reason, it can only adopt directional analog omnidirectional to search, but less dynamic tracking capability; after obtaining the approximate location of destination, it may be partial scan, its dynamic tracking capability better than omnidirectional scanning [2-3]. It will get better results if coupled with the pitch and rocker to mend. According to the mended pitch angle, and rocker angle the antenna always maintain alignment or tracking state.

\subsection{Networking technology}

Due to the topology of the motor transport industry data link network is dynamic, instantaneous [4-5], and with the introduction of the directional antenna, the networking requirements are also increasing. Therefore, the motor transport industry based networking technology data links have gradually become a hot research. Networking technology covers a very wide range, such as: a macro network design, MAC access design to category specific initialization algorithm, routing algorithms, etc. [6-8].

\section{Classified of Network Technology}

Based on peer technology motor transport industry datalink network system may be planar, and may also be hierarchical style. In Planar structure, all network nodes' function and status are equal, there is no bottleneck nodes, and the network is relatively stable, but the coverage of nodes is relatively small. When there are more users, especially they are in the case of transfer, the processing capacity is weak, control overhead, routing often interrupt 
and other shortcomings, so it is mainly for small and medium networks.

In Classified structure, the network is divided into several cluster, each cluster consists of a cluster head node and a plurality of common components. Communication requires a gateway or a distributed gateway node in the completion of the first cluster. Cluster heads and gateways form a virtual backbone. The most advantage of the classification is the network scalability is good, scale is not limited, routing and control is small and easy to implement local mobility management and network synchronization. In order to reduce costs, improve network scalability, classified network management technology can be used to study.

\section{Joint \& Quit Network Simulation Technology}

OPNET Modeler has a wealth of statistics collection and analysis capabilities, you can directly collect performance statistics commonly used network layer parameters, and there are a variety of collection and treatment method of statistical parameters, furthermore you can also collect special network parameters through the underlying network model. OPNET also has a rich chart display and editing function, simulate error and alarming function, all these can be easily prepared and output into simulation report.

\subsection{Initialization network}

In OPNET software, we chose $400 \mathrm{~km} \times 400 \mathrm{~km}$ simulation scenarios, as shown in Figure 1, and made the following plan:

(i) A total of eight vehicles, consisting of two formations, each formation as a cluster. To form a first class communication within the cluster, second communication between the two clusters.

(ii) Number 1-4 truck is a formation, other similar arrangement, until the 2nd fleet. Each smallest number of aircraft fleet node is default to be captain car, No. 2 truck also serves the entire car up-class car. (Where the series captain vehicle and vehicle group does not necessarily correspond to the network after the completion of relationships with primary and secondary network center, the control center and communications center could be separated.)

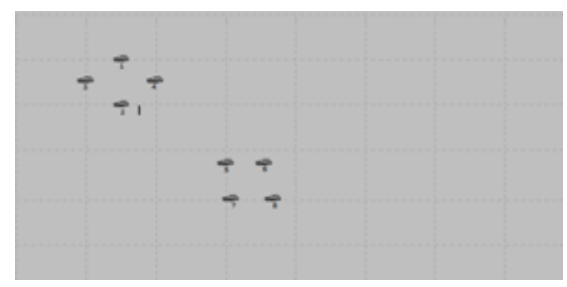

Fig. 1: network initialization simulation scenarios.

\subsection{The new network node of joint \& quit}

In the simulation scenario (Figure 1), we set the node 7 to send a joint network operation every three seconds, Once it successes in jointing than it quit immediately. And wait for the next network joint-time. Network latency curve is shown below in Figure 2.

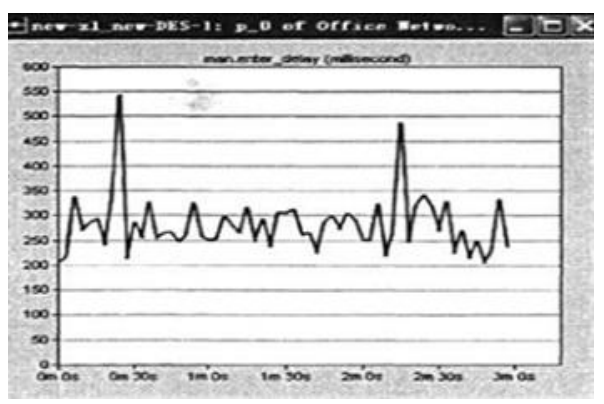

Fig. 2: network initialization simulation results.

2 From the Figure, the majority of the time the network delays are within the scope of the theoretical value. Be able to process network means success. There are still two points more than the theoretical maximum network delay. Analysis of the following two reasons:

One reason: the network node sends a request message network. Ordinary node network message received time slots belong to time-sensitive. Because in OPNET simulations, time-sensitive and network time slots request message using a code word differently, you cannot receive the packet, so in this case ordinary node network cannot successfully received the message. Lead to the first network failure, thereby increasing network latency.

Second reasons: Because of errors in the communication process, may lead to transmission errors, resulting in network failure, thus extending the network delay.

Thus, for the node joint and quit the network and its program design almost meet the requirements of network nodes.

\section{Antenna Simulation Model}

To build a wireless network based on Figure 1 by using OPNET software. This network includes a mobile interference node and two fixed base stations. By configuring the trajectory, the interfering node can be moved, so that the dynamic network topology changes [910] can be observed thus to research impact of this change to the accepted signal quality.

Meanwhile, the mobile node generates interfering radio noise and the noise ratio reduced. In order to improve network performance, directional antenna will be used to enhance the anti-jamming capability of the network. Therefore, the antenna will be used to create a model editor directional antenna models. Finally, we can see through the 
experiment, when the base station uses directional antenna, omnidirectional antenna SNR network has significantly improved.

The simulation results are as follows, mrt_netantenna_test_1 is omnidirectional antenna model and mrt_net-antenna_test_2 is directional antenna models.

Bit error rate of omnidirectional antenna model gradually increased with the interference and the receiver and the transmitter node receiver node distance' decrease. When the interference from the transmitter and receiver minimizes, bit error rate maximizes. But to a certain extent, the error rate will no longer be receiving too much, so there will be a zero appears. After this, as the distance becomes far the reverse process will repeated once. Omnidirectional antenna in the whole simulation process receives the interference signal.
As the figure show: in the beginning, with jammer node and receiver node distance reduce, the error rate increased of Recipient. But with the change of the distance, the vector which connects interference transmitter antenna and receiver antenna is not in the rage of maximum gain. So the receiver node does not accept interference from the interfering transmitter, the error rate is reduced to zero.

As can be seen from the simulation process, the average value of the received packet is dropped. Because there are certain conditions when the antenna receiving the package: that the error rate cannot exceed a certain value. For directional antennas, when direction vector that connects interfering transmitter and receiver is the same as the antenna and the receiver antenna direction of maximum gain, the packet throughput is very low. However, after some time, the package begins to increase acceptance.

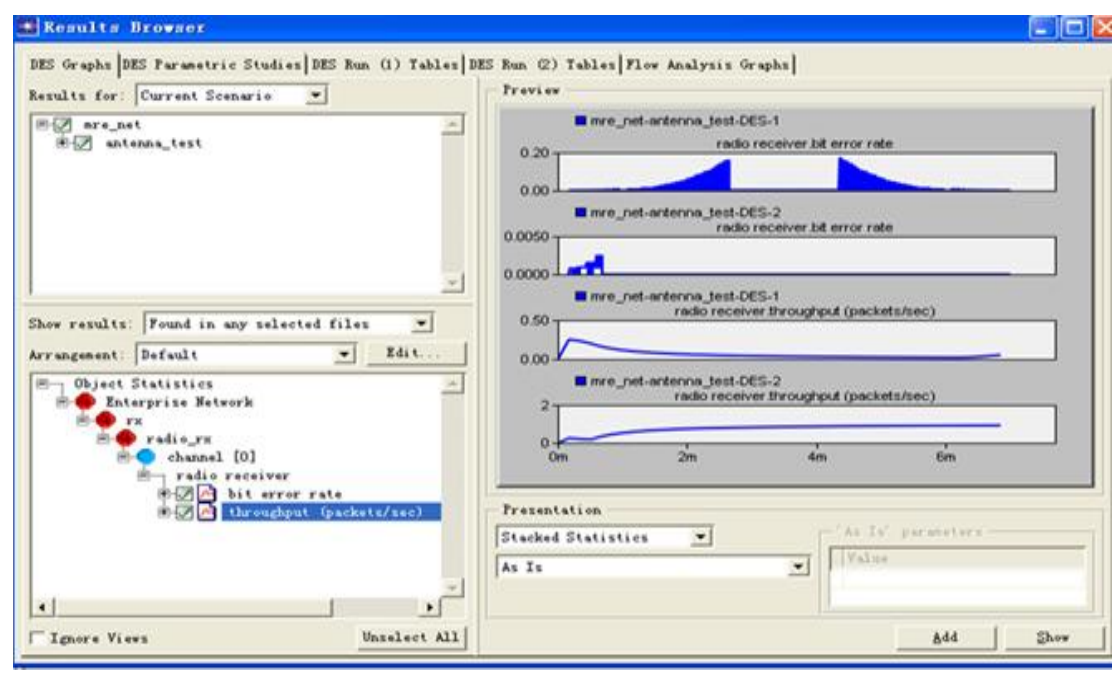

Fig. 3: antenna mode simulation result.

\section{Conclusions}

In this paper, we take practical problem in transportation industry as the starting point and directional antenna and point to point technology network initialization as solution. We put up new joint and quit network method. Finally, we use OPNET Modeler simulation software modeled automobile transportation industry data-link network. We verify the feasibility of the program from theory and simulation technology. The result shows that the design meets the requirements. We believe that with the continuous improvement of the automobile transportation industry data-link, these methods is bound to promote the development of automotive networking technology and have a profound impact on the automotive industry.

\section{References}

[1] K. L. ZHAO, and ZH. L, "Design and Simulation of Multi-Data Link Networking Based on OPNET", Electronics Optics \& Control, vol. 21, pp. 61-64, 2014.

[2] X. H. ZHANG, X. ZH. LIU, SH. Y. LU, and CH. QU, "Research on Wireless Network Experiments Based on OPNET", Journal of Dongguan University of Technology, vol. 20, pp. 63-66, 2013.

[3] ZH. WANG, "Research on the technique of simulation validation based on the directional data link networking", Electronic Design Engineering, vol. 21, pp. 171-174, 2013.

[4] X. Y. ZHANG, "OPNET based cognitive Ad Hoc networks modeling", Microcomputer \& Its Applications, vol. 32, pp. 48-51, 2013.

[5] CH. ZHU, and Y. H. DONG, "Network Simulation Technology and Its Application Based on OPNET", Radio Engineering, vol. 43, pp. 12-15, 2013. 
[6] Z. X. PEI, and X. H. TAN, "A Performance Simulation Study of Train Communication Networks Based on Ethernet", Journal of Southwest University of Science and Technology, vol. 29, pp. 66-71, 2014.

[7] S. G. CHEN, X. JIANG, J. Wang, and Y. H. Liu, "Mashup of vehicular ad-hoc network and universal mobile telecommunications system", Journal of Jilin University (Engineering and Technology Edition), vol. 43, pp. 706-710, 2013.

[8] Y. Q. WANG, J. LI and G. CHEN, "Repeated sending file delivery protocol in satellite networking communication", Journal of Systems Engineering and Electronics, vol. 23, pp. 815-823, 2012.

[9] F. Huang, "OPNET Simulation Technology and Applied Research Network", Network Security Technology \& Application, vol. 1671-0428, pp. 31-33, 2015.

[10]ZH. TANG, and Y. D. WANG, "Simulation of IEEE802.15.4 Wireless Cluster Tree Network Based on OPNET", Journal of Shenyang University of Chemical Technology, vol. 29, pp. 82-87, 2015. 